Respiration 2015;89:435

DOI: $10.1159 / 000371451$

\section{The Use of Propofol for Sedation in Medical Thoracoscopy}

Morné J. Vorster ${ }^{\mathrm{a}}$, Johannes W. Bruwer ${ }^{\mathrm{a}}$, Wolfgang Frank ${ }^{\mathrm{b}}$, Maurizio Bernasconic, Coenraad F. Koegelenberg ${ }^{\text {a }}$

aDivision of Pulmonology, Department of Medicine, Stellenbosch University and Tygerberg Academic Hospital, Cape Town, South Africa; ${ }^{b}$ Lungen-Klinik Amsee, Waren (Müritz), Germany; ${ }^{\mathrm{C}}$ Respiratory Cell Research, University Hospital of Basel, Basel, Switzerland

We read with great interest the paper by Grendelmeier et al. [1] on the randomized trail comparing propofol versus midazolam used for sedation in medical thoracoscopy. The authors not only found the mean lowest oxygen saturation during the procedure to be significantly lower in the propofol group as compared to the midazolam group (93 vs. $96.3 \%, \mathrm{p}=0.007$ ), but also observed more episodes of hypoxaemia ( $27 \mathrm{vs.} 4 \%, \mathrm{p}=0.007)$ and hypotension $(82$ vs. $40 \%, \mathrm{p}<0.0001)$ in the propofol group. They concluded that propofol should not be considered the first choice for sedation in medical thoracoscopy.

We initiated a very comparable multicentre randomized study in 2013, aiming to compare both the safety and adequacy of medical thoracoscopy performed with 2 different conscious sedation regimens (midazolam/fentanyl vs. propofol/fentanyl) administered by a non-specialist anaesthetist. Unlike the study by Grendelmeier et al. [1], propofol was given in 10 - to 20 -mg boluses, targeting an Observer's Assessment of Alertness/Sedation scale of 2-3, and fentanyl was used as an opiate [2]. Procedure time, complications and patient discomfort were defined and documented in a very similar way to the study by Grendelmeier et al. The adequacy of the sedation according to the endoscopist (on a relative scale of 5) and recovery time were also measured, and the criteria for the patients' discharge from the recovery unit were strictly defined [3].

Following the online publication of the study by Grendelmeier et al. [1], the steering committee of our study decided to perform an interim analysis. We enrolled 38 patients (aged $67.5 \pm 11.9$ years, 23 of them males) up to this point, with 18 patients randomised to propofol and 20 to midazolam. We observed no differences in procedure time ( $37.6 \mathrm{vs} .36 .2 \mathrm{~min}, \mathrm{p}=0.57)$, recovery time ( 20.1 vs. $20.8 \mathrm{~min}, \mathrm{p}=0.86$ ), adequacy of sedation as perceived by the endoscopist ( 4.23 vs. $4.15, \mathrm{p}=0.73$ ), patient discomfort or the willingness to undergo another thoracoscopy. There were, however, 10 adverse events observed in the propofol group compared to 4 in the midazolam group $(\mathrm{p}=0.04)$. Adverse events/complications in the propofol group included desaturation responsive to supplementary oxygen $(n=6)$, desaturation requiring temporary bag valve ventilation $(\mathrm{n}=1)$, hypotension requiring intravenous fluid resuscitation $(n=2)$ and the need to abort the procedure $(\mathrm{n}=1)$. Complications seen in the midazolam group included desaturation responsive to supplementary oxygen $(n=3)$ and hypotension not requiring any intervention $(n=1)$. The mean lowest oxygen saturation did not differ between the groups (91.8 vs. $92.7 \%, \mathrm{p}=0.68)$.

Our interim findings therefore support the safety concerns raised by Grendelmeier et al. [1], and we concur that propofol (given either in boluses or as an infusion) should not be considered the drug of choice for sedation during medical thoracoscopy. We have since terminated our study.

Propofol has been shown to be a safe alternative for sedation during flexible bronchoscopy $[4,5]$. In fact, the use of propofol has been shown to be associated with a significantly higher readinessfor-discharge score $1 \mathrm{~h}$ after the examination and a lower proportion of persistent sedation $2 \mathrm{~h}$ after bronchoscopy [4]. One possible explanation for the apparent disparity in its safety profile when used for sedation in thoracoscopy may be related to the procedure itself. Rapid changes in intrapleural pressure due to the introduction of air in the pleural space and/or the aspiration of pleural fluid may cause shunting and ventilation-perfusion mismatching during thoracoscopy, resulting in hypoxaemia and possible hypercarbia. It would seem plausible that propofol blunts the patients' ability to physiologically respond to alterations in the partial pressure of carbon dioxide and oxygen more than midazolam.

There are several limitations to both our study and the study by Grendelmeier et al. [1], including using procedure-related hypoxaemia and hypotension as surrogate markers of morbidity and mortality. Only large multicentre studies with a very structured protocol and follow-up will be able to provide irrefutable evidence on the safety (or lack thereof) of propofol in this setting.

Financial Disclosure and Conflicts of Interest

The authors have no financial disclosures and conflicts of interest to declare.

\section{References}

1 Grendelmeier P, Tamm M, Jahn K, Pflimlin E, Stolz D: Propofol versus midazolam in medical thoracoscopy: a randomized, noninferiority trial. Respiration 2014;88:126-136.

-2 Tschopp J-M, Purek L, Frey J-G, Schnyder J-M, Diaper J, Cartier V, Licker M: Titrated sedation with propofol for medical thoracoscopy: a feasibility and safety study. Respiration 2011;82:451-457.

3 White P, Song D: New criteria for fast-tracking after outpatient anesthesia: a comparison with the modified Aldrete's scoring system. Anesth Analg 1999;88:1069-1072.

4 Stolz D, Kurer G, Meyer A, Chhajed PN, Pflimlin E, Strobel WTM: Propofol versus combined sedation in flexible bronchoscopy: a randomised non-inferiority trial. Eur Respir J 2009;34:1024-1030.

5 Grendelmeier P, Tamm M, Pflimlin E, Stolz D: Propofol sedation for flexible bronchoscopy: a randomised, noninferiority trial. Eur Respir J 2014;43:591-601.

\section{KARGER 125}

C) 2015 S. Karger AG, Base

$0025-7931 / 15 / 0895-0435 \$ 39.50 / 0$ 